\title{
Monitoring Fatigue Status in Elite Team-Sport Athletes: Implications for Practice
}

\author{
Robin T. Thorpe, Greg Atkinson, Barry Drust, and Warren Gregson
}

\begin{abstract}
The increase in competition demands in elite team sports over recent years has prompted much attention from researchers and practitioners to the monitoring of adaptation and fatigue in athletes. Monitoring fatigue and gaining an understanding of athlete status may also provide insights and beneficial information pertaining to player availability, injury, and illness risk. Traditional methods used to quantify recovery and fatigue in team sports, such as maximal physical-performance assessments, may not be feasible to detect variations in fatigue status throughout competitive periods. Faster, simpler, and nonexhaustive tests such as athlete self-report measures, autonomic nervous system response via heart-rate-derived indices, and to a lesser extent, jump protocols may serve as promising tools to quantify and establish fatigue status in elite team-sport athletes. The robust rationalization and precise detection of a meaningful fluctuation in these measures are of paramount importance for practitioners working alongside athletes and coaches on a daily basis. There are various methods for arriving at a minimal clinically important difference, but these have been rarely adopted by sport scientists and practitioners. The implementation of appropriate, reliable, and sensitive measures of fatigue can provide important information to key stakeholders in team-sport environments. Future research is required to investigate the sensitivity of these tools to fundamental indicators such as performance, injury, and illness.
\end{abstract}

Keywords: training, performance, wellness, recovery, injury, illness

Elite team-sport athletes, particularly those in the professional football codes, are exposed to high competition loads, particularly in recent years. These high loads reflect a number of factors, including an increased frequency of domestic competitions, particularly for higher-level athletes, as well as a higher intensity of competition due to enhanced player preparation strategies. ${ }^{1}$ Higher loads may also result from the increased demands of international competition during both the domestic season and the off-season period.

An increased availability of athletes for selection, as a result of a reduction in injuries, substantially increases a team's chance of success. ${ }^{2}$ Therefore, injury prevention strategies are fundamental to the work of the athlete's support team. Routine modifications in training load (frequency, duration, intensity) occur during the training cycle and these subsequently increase or decrease fatigue. Management of fatigue is important in mediating adaption to training and ensuring the athlete is prepared for competition, ${ }^{3}$ as well as for reducing the athletes' susceptibility to nonfunctional overreaching, injury, and illness. ${ }^{4}$

The importance of managing athlete fatigue has led to an increase in interest in monitoring athlete loads, particularly in terms of the measures which may offer insights into whether the athlete is adapting positively or negatively to the collective stresses of training and competition. In the present review, we will consider published research concerned with the monitoring of fatigue status in team-sport athletes. Information derived from other sports will be examined where evidence in team-sport settings is not available.

Thorpe is with the Medicine and Science Dept, Manchester United, Manchester, UK. Atkinson is with the Health and Social Care Inst, Teesside University, Teesside, UK. Drust and Gregson are with the Research Inst for Sport and Exercise Sciences, Liverpool John Moores University, Liverpool, UK. Address author correspondence to Robin Thorpe at robin. thorpe@manutd.co.uk.
While there are studies in which various proposed moderators and mediators have been found to be statistically significantly associated with fatigue status, in our paper we also highlight the various measurement issues and practical considerations which should be considered by those responsible for the development and implementation of player monitoring systems in the field. We focus, especially, on the neglected topic of all the different approaches to selecting a minimal worthwhile change in fatigue status. It is hoped that our overview will provide the basis for the development of a framework for evaluating fatigue status in team sports and provide some guidance for future investigators.

It is not our intention to comprehensively describe the available information on the etiology of fatigue or the scientific basis of common recovery intervention practices. Such information can be found in several excellent recent reviews. ${ }^{5,6}$ For the purpose of this review, and to align with previous reviews in this area, fatigue will be defined as an inability to complete a task that was once achievable within a recent time frame..$^{3,7}$

\section{Methods for Monitoring Fatigue}

Training load reflects the internal and external loads imposed upon the athlete. ${ }^{8}$ External load relates to work completed by the athlete independent of his or her internal characteristics and is important for understanding the capabilities and capacities of the athlete. ${ }^{7}$ The internal load, or the relative physiological strain resulting from the external training factors, is also crucial to determining both the stress imposed and subsequent adaptation to training. ${ }^{9}$ A combination of both the external and internal load is therefore important for training since the uncoupling or divergence of external and internal loads may differentiate between a nonfatigued and a fatigued athlete. ${ }^{3,7}$ This approach is particularly relevant in "closed loop" sports like cycling where the performance outcome is time, and the power produced by the rider is known to have a relatively 
precise association with the performance time. Under such conditions, the internal load needed to sustain a certain external load (power output) can provide important information regarding the athlete's fatigue status. ${ }^{7}$

In contrast to closed-loop sports, the ability to relate external and internal loads in open-loop sports like team sports is difficult due to the inherent variability in physical performance during sport-specific training drills ${ }^{10}$ and match play. ${ }^{11}$ As a consequence, attempts to monitoring the fatigue status of team-sport athletes have largely focused on the assessment of internal and external load measures under resting conditions and/or during submaximal exercise assessments on the morning prior to training. Within the confines of this approach, a valid indicator of fatigue in team sports should be sensitive to training load and their response to acute exercise should be distinguishable from chronic changes in adaptation. ${ }^{12}$ Prospective tools should also be non-invasive, quick and easy to administer and limit any additional loading on the athlete. This is particularly important in football codes, where competition occurs on a weekly basis and in some instances 2 or 3 times a week, meaning that players are required to peak with limited recovery between matches.

\section{Athlete Self-Report Measures}

Recent surveys on fatigue monitoring in high-performance sport demonstrate that athlete self-report measures (ASRM) are used extensively for assessing the overall well-being of team-sport athletes. ${ }^{13}$ A plethora of ASRM currently exist including the POMS, ${ }^{14,15}$ DALDA, ${ }^{16} \mathrm{TQR},{ }^{17}$ and REST-Q ${ }^{18,19}$ which have been extensively documented in the literature. However, many of these are often extensive and time-consuming to complete preventing their use on a daily basis with large numbers of team athletes. Many team sports therefore often adopt shorter, customized questionnaires which can be administered on daily basis. ${ }^{13}$ A recent review highlighted that ASRM demonstrate greater sensitivity to acute and chronic training loads than commonly used objective measures. ${ }^{20}$ In team sports, for example contemporary Australian Football League (AFL) and English Premier League (EPL) research has shown custom psychometric scales to be sensitive to daily, within-weekly and seasonal changes in training load. ${ }^{21-23}$ Indeed, daily ASRM (fatigue, sleep quality, stress, mood, and muscle soreness) were significantly correlated with daily training load in a pre-season camp and competitive period in AFL and EPL players, respectively. ${ }^{22,24}$ Similarly, ASRM were sensitive to changes in training load during typical weeks in AFL and EPL players across the course of the season. ${ }^{21,23,25}$ Further importance of ASRM and relationship with injury/illness has been observed in Rugby League, in this study fluctuations in ASRM between macrocycles were shown to provide useful insights into possible illness risk in players. ${ }^{26}$ Further work is required to examine the relationships between ASRM and injury/illness risk in team-sport athletes.

\section{Autonomic Nervous System}

The autonomic nervous system (ANS) is interlinked with many other physiological systems, ${ }^{27}$ significant attention in the literature has therefore centered upon the use of indictors of ANS functioning for determining an athletes overall adaptation/fatigue status. To date this has largely stemmed from studies examining the sensitivity of heart-rate (HR) -derived indices including resting HR (RHR), exercising HR (HRex), ${ }^{28} \mathrm{HR}$ variability (HRV), ${ }^{27,28}$ and HR recovery $(\mathrm{HRR})^{28,29}$ to fluctuations in training and competition load.

\section{Submaximal Heart Rate}

Decreases in HR during standardized exercise bouts have traditionally been associated with increases in aerobic fitness. However, the majority of data available has reported inconsistent results in non-team-sport athletes. Heart rate during intensified training and during varying intensities showed significant reductions in overreached triathletes. Le Meur et $\mathrm{al}^{28,30,31}$ suggested a hyperactivation of the parasympathetic nervous system via central, cardiac and/ or periphery mechanisms. Recent observations in AFL have also reported reductions in heart rate in response to training during preseason, although, the authors concluded this was more possibly due to the effects of training/environmental induced changes in plasma volume than acute changes in fitness or fatigue. Contrary to these reports, exercising heart rate in EPL players failed to fluctuate in response to within week changes in training and match load over the course of a season. ${ }^{25}$ The use of HRex in healthy athletes to predict negative effects in performance or fatigue should be treated with caution and interpreted together with other potential measures of fatigue such as ASRM. ${ }^{28,32}$

\section{Heart-Rate Variability}

Vagal-related time domain parameters of HRV have recently received greater attention than more traditional spectral analyses due to their superior reliability and assessment capture over short periods of time. ${ }^{33,34}$ Sensitivity to changes in training load and performance has mainly been observed in non-team sports. ${ }^{27,35}$ Generally, HRV is reduced (sympathetic dominance) in the immediate days after intense exercise, ${ }^{36}$ however, results from endurance sports have shown inconsistent results. ${ }^{37}$ Little evidence currently exists with regard to its sensitivity to fluctuations in training and competition load in team sports. In AFL players undertaking preseason training in the heat, a vagal-related HRV parameter (SD1) was largely and statistically significantly correlated $(r=\sim .5)$ to daily RPE-TL. ${ }^{22}$ However, these unexpected changes in parasympathetic activity may have been partly mediated through thermoregulatory mechanisms associated with alterations in plasma volume. ${ }^{22,36}$ In elite soccer, HRV (Ln rMSSD) appeared to decrease $(r=-.2)$, albeit transiently, in response to high-speed-running distance. ${ }^{24}$ Contrastingly, in the same population, HRV did not change across a standard in-season training week. ${ }^{25}$ Interestingly, data derived from endurance sports have suggested that the sensitivity of HRV to training and competition may be improved when data is averaged over a week or using 7-day rolling averages compared to the use of single data points due to the high day-to-day variation in these indices. However, undertaking such measures may prove difficult with the large volume of athletes engaged in team sports. ${ }^{38}$ Future research is needed to determine whether such approaches enhance the suitability of these measures for use in team-sport populations.

\section{Heart-Rate Recovery}

Postexercise HRR reflects general hemodynamic adjustments in relation to body position, blood pressure regulation, and metaboreflex activity, which partly drives sympathetic withdrawal and parasympathetic reactivation. ${ }^{39}$ Recent findings in endurance sports have shown that HRR may serve as a sensitive marker of acute training-load alteration, ${ }^{27,29}$ although this association has yet to be seen in team sports. ${ }^{32} \mathrm{HRR}$ did not fluctuate in response to daily and within-week training-load variability in EPL players. ${ }^{24,25}$ Data from physically active men and women have shown a delay in HRR following increases in training load. ${ }^{27}$ More recently, nonfunction- 
ally overreached elite triathletes showed a faster ( 8 beats/min) HRR than elite triathlete controls after the same training program. ${ }^{40} \mathrm{It}$ appears that HRR is responsive to both acute and chronic changes in training load; however, the exact direction of this change and whether HRR can detect fatigue status remains unclear and should be interpreted alongside training status and with caution. ${ }^{41}$

\section{Physical Performance}

A variety of maximal-performance assessments (sprints, repeated sprints, jumps, and maximal voluntary contractions) have been used in attempt to quantify the rate of recovery of performance in the hours and days after training and competition in team sports. ${ }^{42-46}$ While these types of assessment provide important information, the application of physical performance tests which are exhaustive in nature and time-consuming to deliver means they are often unsuitable for use in team-sport environments. ${ }^{46,47}$ Being fast, efficient, and without additional load represent the only feasible maximalperformance assessments applicable for team-sport players.

\section{Neuromuscular Function}

Various jump protocols including squat jump (SJ) and countermovement jump (CMJ) have been adopted to examine the recovery of neuromuscular function after competition with significant decreases for up to 72 hours routinely reported. ${ }^{42-46}$ However, less attention has focused on examining their sensitivity to changes in training load. CMJ was not sensitive as a measure of neuromuscular status in EPL players when analyzed alongside daily fluctuations in training load, furthermore, data derived from elite rugby sevens and adolescent soccer players revealed no change in countermovement jump height or correlation to training load during a taper and across a training period respectively. ${ }^{48-50}$ The use of jump height per se as a global indicator of neuromuscular function may lack the sensitivity to detect changes in training load in previous studies. Moreover, $\mathrm{CMJ}$ height alone may mask alternative neuromuscular measures and their sensitivity to alterations in load. Reductions in 18 different neuromuscular variables were found after a high-intensity fatiguing protocol in college-level team-sport athletes. ${ }^{51}$ Neuromuscular parameters (eccentric, concentric, and total duration, time to peak force/power, flight time:contraction-time ratio) derived from $\mathrm{CMJ}$ were deemed suitable for neuromuscular fatigue detection. ${ }^{51}$ In AFL, variations in force-time parameters (flight time:contraction-time ratio) were observed over the course of a season, indicating sensitivity to increases in load over time. ${ }^{52}$ Future research is required to investigate whether alternative measures derived from CMJ are sensitive to changes in training load in elite team-sport athletes.

\section{Joint Range of Motion/Flexibility}

Simple clinical assessments of joint range of motion (JROM) have been typically performed on a one-off basis as part of a preseason screening battery in elite team sport. ${ }^{53}$ However, there is a lack of data reporting JROM responses to training and match load. Indeed, the assessment of JROM more regularly during competitive periods may provide greater information pertaining to structural fatigue and potential injury risk compared to a single pre-season assessment. In elite soccer players, knee range of motion was reduced until 48 hours postmatch. ${ }^{43}$ Similarly, Mohr et al ${ }^{54}$ found knee-joint range of motion declined $7 \%$ at both 24 and 48 hours postmatch. Moreover, structural assessments quantifying hip/groin extensibility have shown good reliability and validity after match play in youth soccer players. ${ }^{55}$ Indeed, a reduction of more than $12.5 \%$ in adductor (bent-knee fall-out test) range 14 hours postmatch was deemed a meaningful change in youth soccer players. The simple and quick nature of JROM assessments evaluating key anatomical regions may provide a greater understanding of structural status and potential injury risk. Future research is required, to examine the time-course of recovery for JROM measures postmatch and their sensitivity to changes in load in team-sport athletes.

\section{Biochemical/Hormonal/Immunological}

A large amount of research has examined a range of biochemical, hormonal and immunological responses to team-sport competition. ${ }^{46,47}$ It is beyond the scope of this article to review the collective literature surrounding the responses of such measures in team sports, however, no definitive marker has been derived for examining the fatigue status of athletes. Furthermore, the associated costs and in some instances time consuming nature of their analyses, often means many of these measures are impractical for use in the teamsport environment.

A variety of markers have been used in an attempt to examine potential levels of muscle damage in athletes. Creatine kinase (CK) increases immediately postmatch in soccer ${ }^{43}$ and rugby ${ }^{56}$ and peaks between 24 and 48 hours with a return to baseline values observed from 48 to 120 hours. ${ }^{42-46,57}$ Although widely used, questions remain regarding the exact mechanism of activity following exercise and its relationship with muscle function recovery. ${ }^{46,58} \mathrm{IL}-6$ is produced in larger amounts than any other cytokine prompting its use as a global measure of inflammation. ${ }^{59} \mathrm{IL}-6$ peaks immediately after the cessation of exercise and then rapidly returns to baseline values after 24 hours. ${ }^{42,43,54} \mathrm{C}$-reactive protein (CRP) and uric acid have been found to be a more sensitive marker of inflammation after soccer match play. ${ }^{43,45,54}$ Indeed, increases of up 50\% 48 hours post match have been observed in elite soccer players. ${ }^{45}$ Furthermore, in a similar study in elite soccer players, uric acid peaked 72 hours after a match. ${ }^{43}$ Adrenal hormones cortisol and testosterone have been shown to increase up to 48 hours after competition and up to $50 \%$ postcompetition in team sports, respectively. ${ }^{54}$ Salivary immunoglobulin A ( $\mathrm{S}-\operatorname{Ig} \mathrm{A})$ has become a popular means to assess mucosal immunity in athletes via the use of real-time lateral flow devices. In EPL players S-IgA showed reductions during a taper phase and a period of international competition, ${ }^{60,61}$ Little longitudinal data, particularly around competition and training phases, currently exist in team sports. The impractical nature and cost of individual samples may explain the limited data assessing biochemical, hormonal, and immunological measures over extended training and competition periods in team sports.

\section{Measurement Considerations}

In a recent British Journal of Sports Medicine editorial, McCall et al ${ }^{62,63}$ described the concept of "working fast-working slow," whereby the researcher undertakes robust and sometimes longterm studies in order for the practitioner to make informed, sometimes "on the spot," decisions in conjunction with players and coaches. This working fast-working slow concept is a relevant measurement issue because practitioners need to ensure that a given measurement of fatigue or performance can be interpreted quickly and accurately against the backdrop of random withinsubject variability, which may be quantified by the researcher in 
a well-designed reliability study. Any measurement of fatigue also needs to be interpreted with knowledge about how valid the measurement tool is to the ultimate outcomes of player performance, illness, and/or injury. This knowledge is derived from well-designed and robust validity studies, ${ }^{64}$ as well as prognostictype studies in which fatigue is the predictor and injury rate, for example, is the outcome.

Measurement decisions should definitely not be based solely on whether a particular reliability or validity statistic, for example, a correlation coefficient, is "statistically significant" or not. ${ }^{64}$ Similarly, published general qualitative thresholds of excellent, good, or moderate measurement statistics may not always be fully informative for decision-making purposes. For example, it has been thought in the past that a measurement tool can be deemed sufficiently reliable if the test-retest coefficient of variation (CV) is $<10 \%$ and/or if a test-retest correlation is $>.8$, but the rationale for these general thresholds has not been very clearly described in the past. ${ }^{64}$ The ideal process is for a given measurement of fatigue status to be interpreted relative to both random within-subject variability (thereby deriving the "minimal detectable change"), as well as the minimum value that has been deemed to be clinically or practically important, the MCID ${ }^{65-67}$ It is important to consider that the minimal detectable change might not be the same as the MCID. A minimum detectable change is the smallest true change that has a reasonable probability of being statistically significant. A properly powered study should mean that the minimum detectable effect is achieved, but this effect size might still not be clinically or practically important.

Once the MCID has been selected and reliability/validity information is collected on a relevant sample, this process is essentially probabilistic in nature, whereby one can estimate the chances that a given measurement exceeds the MCID. While useful spreadsheets exist to undertake this process ${ }^{68}$ the magnitude of the selected MCID is critical for the process of monitoring fatigue to be useful and informative in the field.

The quantification of an MCID is also imperative for accurate a priori sample-size estimations in applied research. ${ }^{69}$ This samplesize estimation is important for ethical and economic reasons. It may be unethical and wasteful of time and money for a researcher to collect data, possibly with invasive methods, if the sample size is too small to detect the MCID. A large sample size may also be unethical if a treatment effect could have been quantified with adequate precision using a much smaller sample.

An MCID might be relatively straightforward to select in closed-loop sports like individual track cycling where the performance outcome to anchor to is clearly time, and objective indicators like the power produced by the rider are known to have a relatively precise association with this performance time. Changes in performance time (and associated indicators) may also be stable enough for the practitioner to link directly to meaningful changes in athlete ranking. ${ }^{68}$ Nevertheless, the selection of an MCID for team-sport performance is more difficult due to the multicomponent nature of these sports and the relatively high within-subject variability in physical performance between successive soccerspecific training drills ${ }^{10}$ and match play. ${ }^{11,70}$ Furthermore, the use of highly demanding maximal-performance tests, as possible closer surrogates of match performance, is difficult in team sports due to the limited recovery time between frequent competitions and matches. The magnitude of an MCID may also depend on whether it is considered from the perspective of the athlete, the coach, or even the club's financers/owners. For example, an MCID for a player may be the change in fatigue that leads to a perceived increase in match performance. The coach may be more interested in the MCID that confers the greatest probability of longer-term availability and the financers may be more interested in the MCID that confers both the former consequences, as well as adding to the transfer value of the player. Obviously, all of these anchors are associated with each other, yet it is uncommon for these different perspectives to be considered together, using a Delphi method, for example.

The approaches for selection of an MCID have mostly been considered in detail from a clinical trial perspective. ${ }^{66,67,71}$ Researchers and practitioners could gather information about the MCID using expert/end-user opinion, evidence synthesis and a pilot study, ideally by triangulating across these different approaches. Nevertheless, although a certain difference derived from an evidence synthesis or pilot study might be a realistic target, it might not necessary be clinically/practically important itself.

There are 2 main types of approaches for quantifying an MCID; anchor and distributional (statistical). In the "anchor" approach, the measurement (or change in measurement) is anchored to an associated change in another external measure of change, the anchor variable. For example, a change in fatigue status could be anchored to changes in illness, soft-tissue injury, and/or match performances/ rankings. Ideally, the robustness of any relationship between an indicator variable (or exposure) and the anchor should be quantified in prognostic-longitudinal type research. ${ }^{72}$ In these types of studies, it is imperative to employ the most appropriate analysis approaches, as highlighted recently by Finch and Marshall. ${ }^{73}$ These authors recommended that epidemiological studies into sports injuries should use the "subsequent injury categorization" model to make full use of all the longitudinal data that are collected.

Distributional approaches for specifying an MCID can be based on the standard error of measurement (typical error) and/ or the between-subjects SD for the measured variable (eg, >0.2 $\mathrm{SDs}$ ). In the former approach, a measurement (or change in measurement) is compared with an MCID that is larger than the random and unavoidable within-subject variability (standard error of mean). Additional decisions in this approach are how much larger than random variability should the MCID be, as well as what level of statistical precision should be selected, although it has been reported that a reasonable MCID approximates to $1 \mathrm{SEM}^{74}$

A related concept to the SEM is the minimal detectable change. For example, a change in measurement could be deemed important if it exceeds twice the standard deviation of differences (derived from a reliability study). The standard deviation of differences can be estimated by multiplying the SEM by the square root of 2 . This threshold of 2 SDs is essentially the $95 \%$ limits of agreement described by Bland and Altman. ${ }^{75,76}$

Health economics may also be factored into the MCID selection process. This would involve defining a threshold value for the cost of a change in performance or a reduction in illness or injury that a coach or team owner is willing to pay. Then data on the differences in costs, effects and harms can be all considered together to arrive at an estimate of relative efficiency. It is common for changes in measurements to be considered on a standardized scale, for example, as a given fraction of the between-subjects standard deviation. Various thresholds have been proposed for trivial, small, medium, and large effects. ${ }^{77,78}$ For injury applications, binary or survival (time-to-event) outcome metrics (eg, an odds, risk, or hazard ratio) can be considered in a similar way. Effect-size thresholds for risk ratio values depend on the "nominal" event proportion, that is, that observed in the control group. 


\section{Practical Applications and Future Direction}

Elite team-sport athletes compete on a weekly basis and often 2 or 3 times per week in the football codes. While adhering to the fundamental measurement requirements outlined in this review, prospective fatigue-monitoring tools should also be noninvasive and time-efficient due to the large volume of players who may require assessing on any given day. The tools should also minimize any additional loading due to the limited recovery time available to team-sport athletes during the competition phase. Recent surveys on current trends of fatigue monitoring in high performance sport, highlight athlete self-report questionnaires as the most frequently adopted tool, particularly customized designs consisting of 4 to 12 items. ${ }^{13}$ The validity of these tools is also supported by a number of reports highlighting their sensitivity to training and/or match load. ${ }^{21,22,25,79,80}$ However, the efficacy of these tools depends on a number of theoretical (interrelations between the measure, social environment, and outcomes) and practical factors that need to be addressed in the applied sport setting. ${ }^{20}$

Research to date has mainly examined the sensitivity of prospective fatigue-monitoring tools to previous training and match load. Future work is required to examine the degree to which a fatigue measure or change in fatigue measure promotes subsequent changes in a relevant anchor such as performance, illness, and injury. This represents a move towards establishing the MCID for fatigue measures and in doing so align with approaches adopted in clinical practice ${ }^{71}$ as outlined earlier in this review. From a performance perspective, establishing pretraining or prematch MCID for fatigue measures would theoretically offer an indication on the quality of the external output that might be produced. ${ }^{23}$ This would provide coaches with the ability to make adjustments to the scheduled training or rotate the players from a match perspective. However, application to match play is unlikely to be feasible due to the high inherent variability of match-play physical performance in team sports. ${ }^{11,70}$ The use of more closed-loop sport-specific training drills where players may possibly perform more consistent physical outputs may afford an opportunity to establish pre-training MCID for fatigue measures.

There is now a growing body of literature highlighting links between increased athlete loading (competition and training loads) and the incidence of soft-tissue injury and, to a lesser extent, illness in team sports (see Drew et $\mathrm{al}^{81}$ for a review of recent work). Furthermore, internal loading may offer more accurate predictions of injury risk than measures typically used to estimate the external load. ${ }^{7}$ In these studies, internal load was largely estimated by multiplying total training or match session duration with session ratings of perceived exertion. ${ }^{82}$ Future work is required to examine whether other measures of the internal load/fatigue status of the athlete may further enhance the precision of these estimates. For example, a number of studies have examined the relationship between psychological stress and injury in sports, ${ }^{83,84}$ although few reports have examined these relationships in team sports using athlete self-report assessments commonly used in practice. ${ }^{85,86}$ By examining the role of various prospective measures of fatigue status we may enhance our ability to observe changes in the athletes status, which may predispose them to illness and/or injury. Although, fatigue experienced by team-sport athletes is multifactorial in nature and a single measure is insufficient in explaining athlete status. A combination of subjective and objective measures is, therefore, more likely in order to quantify fatigue status in elite team-sport athletes.

\section{Summary}

Considering the increase in competition demands in elite team sports over recent years, the quantification of fatigue status has gained popularity among researchers and practitioners. Since maximal physical performance assessments (sprints, repeated sprints, and maximal voluntary contractions) traditionally used to quantify recovery and fatigue in athletes are unsuitable in team-sport environments due to their exhaustive and time-consuming nature, recent literature has demonstrated that quick, simple and noninvasive tools such as ASRM, ANS HR-indices, JROM, and jump protocols that have been shown to be sensitive to changes in training load. Practitioners using such measures must consider the MCID when interpreting results to identify true sensitivity in athlete fatigue status and in turn, informed decisions alongside key stakeholders in elite team-sport environments. This review has outlined the potential measures which may be used as a starting point by practitioners to monitor fatigue status in team-sport athletes, however, future work is required to investigate the relationships between these measures of fatigue and global anchors such as performance, injury, and illness.

\section{References}

1. Bradley PS, Sheldon W, Wooster B, Olsen P, Boanas P, Krustrup P. High-intensity running in English FA Premier League soccer matches. J Sports Sci. 2009;27(2):159-168. doi:10.1080/02640410802512775 PubMed

2. Hägglund M, Waldén M, Magnusson H, Kristenson K, Bengtsson $\mathrm{H}$, Ekstrand J. Injuries affect team performance negatively in professional football: an 11-year follow-up of the UEFA Champions League injury study. Br J Sports Med. 2013;47(12):738-742. doi:10.1136/ bjsports-2013-092215 PubMed

3. Pyne DB, Martin DT. Fatigue insights from individual and team sports. In: Marino FE, ed. Regulation of Fatigue in Exercise. New York: Nova Science; 2011.

4. Nimmo MA, Ekblom B. Fatigue and illness in athletes. J Sports Sci. 2007;25(Suppl 1):S93-S102. doi:10.1080/02640410701607379 PubMed

5. Nédélec M, McCall A, Carling C, Legall F, Berthoin S, Dupont G. Recovery in soccer : part II-recovery strategies. Sports Med. 2013;43(1):9-22. doi:10.1007/s40279-012-0002-0 PubMed

6. Gill ND, Beaven CM, Cook C. Effectiveness of post-match recovery strategies in rugby players. Br J Sports Med. 2006;40(3):260-263. doi:10.1136/bjsm.2005.022483 PubMed

7. Halson SL. Monitoring training load to understand fatigue in athletes. Sports Med.2014;44(Suppl 2):S139-S147. doi:10.1007/s40279-0140253-z PubMed

8. Esposito F, Impellizzeri FM, Margonato V, Vanni R, Pizzini G, Veicsteinas A. Validity of heart rate as an indicator of aerobic demand during soccer activities in amateur soccer players. Eur J Appl Physiol. 2004;93(1-2):167-172. doi:10.1007/s00421-004-1192-4 PubMed

9. Viru AM, Hackney AC, Välja E, Karelson K, Janson T, Viru M. Influence of prolonged continuous exercise on hormone responses to subsequent exercise in humans. Eur J Appl Physiol. 2001;85(6):578-585. doi:10.1007/s004210100498 PubMed

10. Gaudino P, Iaia FM, Alberti G, Hawkins RD, Strudwick AJ, Gregson W. Systematic bias between running speed and metabolic power data in elite soccer players: influence of drill type. Int J Sports Med. 2014;35(6):489-493. doi:10.1055/s-0033-1355418 PubMed

11. Gregson W, Drust B, Atkinson G, Salvo VD. Match-to-match variability of high-speed activities in Premier League soccer. Int J Sports Med. 2010;31(4):237-242. doi:10.1055/s-0030-1247546 PubMed 
12. Meeusen R, Duclos M, Foster C, et al. Prevention, diagnosis, and treatment of the overtraining syndrome: joint consensus statement of the European College of Sport Science and the American College of Sports Medicine. Med Sci Sports Exerc. 2013;45(1):186-205. doi:10.1249/MSS.0b013e318279a10a PubMed

13. Taylor K, Chapman D, Cronin J, Newton M, Gill N. Fatigue monitoring in high performance sport: a survey of current trends. J Aust Strength Cond. 2012;20:12-23.

14. Raglin JS, Morgan WP. Development of a scale for use in monitoring training-induced distress in athletes. Int J Sports Med. 1994;15(2):8488. doi:10.1055/s-2007-1021025 PubMed

15. Buchheit M. Sensitivity of monthly heart rate and psychometric measures for monitoring physical performance in highly trained young handball players. Int J Sports Med. 2015;36(5):351-356. doi:10.1055/s-0034-1385882 PubMed

16. Coutts AJ, Slattery KM, Wallace LK. Practical tests for monitoring performance, fatigue and recovery in triathletes. J Sci Med Sport. 2007;10(6):372-381. doi:10.1016/j.jsams.2007.02.007 PubMed

17. Kenttä G, Hassmén P. Overtraining and recovery: a conceptual model. Sports Med. 1998;26(1):1-16. PubMed doi:10.2165/ 00007256-199826010-00001

18. Kellmann M. Preventing overtraining in athletes in high-intensity sports and stress/recovery monitoring. Scand J Med Sci Sports. 2010;20(Suppl 2):95-102. doi:10.1111/j.1600-0838.2010.01192.x PubMed

19. Coutts AJ, Reaburn P. Monitoring changes in rugby league players' perceived stress and recovery during intensified training. Percept Mot Skills. 2008;106(3):904-916. doi:10.2466/pms.106.3.904-916 PubMed

20. Saw AE, Main LC, Gastin PB. Monitoring the athlete training response: subjective self-reported measures trump commonly used objective measures: a systematic review. Br J Sports Med. 2015. doi:10.1136/ bjsports-2015-094758

21. Gastin PB, Meyer D, Robinson D. Perceptions of wellness to monitor adaptive responses to training and competition in elite Australian football. J Strength Cond Res. 2013;27(9):2518-2526. doi:10.1519/ JSC.0b013e31827fd600 PubMed

22. Buchheit M, Racinais S, Bilsborough JC, et al. Monitoring fitness, fatigue and running performance during a pre-season training camp in elite football players. J Sci Med Sport. 2013;16(6):550-555. doi:10.1016/j.jsams.2012.12.003 PubMed

23. Gallo TF, Cormack SJ, Gabbett TJ, Lorenzen CH. Self-reported wellness profiles of professional Australian football players during the competition phase of the season. J Strength Cond Res. 2017;31(2):495-502. doi:10.1519/JSC.0000000000001515 PubMed

24. Thorpe RT, Strudwick AJ, Buchheit M, Atkinson G, Drust B, Gregson W. Monitoring fatigue during the in-season competitive phase in elite soccer players. Int J Sports Physiol Perform. 2015;10(8):958-964. doi:10.1123/ijspp.2015-0004 PubMed

25. Thorpe RT, Strudwick AJ, Buchheit M, Atkinson G, Drust B, Gregson W. Tracking morning fatigue status across in-season training weeks in elite soccer players. Int J Sports Physiol Perform. 2016;11(7):947-952. doi:10.1123/ijspp.2015-0490 PubMed

26. Thornton HR, Delaney JA, Duthie GM, et al. Predicting Self-reported illness for professional team-sport athletes. Int J Sports Physiol Perform. 2016;11(4):543-550. PubMed http://dx.doi.org/10.1123/ ijspp.2015-0330

27. Borresen J, Lambert MI. Changes in heart rate recovery in response to acute changes in training load. Eur J Appl Physiol. 2007;101(4):503511. doi:10.1007/s00421-007-0516-6 PubMed

28. Buchheit M. Monitoring training status with HR measures: do all roads lead to Rome? Front Physiol. 2014;5:73. doi:10.3389/ fphys.2014.00073 PubMed
29. Lamberts RP, Swart J, Capostagno B, Noakes TD, Lambert MI. Heart rate recovery as a guide to monitor fatigue and predict changes in performance parameters. Scand J Med Sci Sports. 2010;20(3):449-457. doi:10.1111/j.1600-0838.2009.00977.x PubMed

30. Le Meur Y, Pichon A, Schaal K, et al. Evidence of parasympathetic hyperactivity in functionally overreached athletes. Med Sci Sports Exerc. 2013;45(11):2061-2071. doi:10.1249/MSS.0b013e3182980125

31. Le Meur Y, Hausswirth C, Natta F, Couturier A, Bignet F, Vidal PP. A multidisciplinary approach to overreaching detection in endurance trained athletes. J Appl Physiol. 2013;114(3):411-420. doi:10.1152/ japplphysiol.01254.2012 PubMed

32. Buchheit M, Simpson MB, Al Haddad H, Bourdon PC, MendezVillanueva A. Monitoring changes in physical performance with heart rate measures in young soccer players. Eur J Appl Physiol. 2012;112(2):711-723. doi:10.1007/s00421-011-2014-0 PubMed

33. Esco MR, Flatt AA. Ultra-short-term heart rate variability indexes at rest and post-exercise in athletes: evaluating the agreement with accepted recommendations. J Sports Sci Med. 2014;13(3):535-541. PubMed

34. Al Haddad H, Laursen PB, Chollet D, Ahmaidi S, Buchheit M. Reliability of resting and postexercise heart rate measures. Int $J$ Sports Med. 2011;32(8):598-605. PubMed doi:10.1055/s-0031-1275356

35. Manzi V, Castagna C, Padua E, et al. Dose-response relationship of autonomic nervous system responses to individualized training impulse in marathon runners. Am J Physiol Heart Circ Physiol. 2009;296(6):H1733-H1740. doi:10.1152/ajpheart.00054.2009 PubMed

36. Stanley J, Peake JM, Buchheit M. Cardiac parasympathetic reactivation following exercise: implications for training prescription. Sports Med. 2013;43(12):1259-1277. doi:10.1007/s40279-013-0083-4 PubMed

37. Plews D, Laursen P, Kilding A, Buchheit M. Heart-rate variability and training-intensity distribution in elite rowers. Int $J$ Physiol Perform. 2014;9(6):1026-1032. http://dx.doi.org/10.1123/ijspp.2013-0497

38. Plews DJ, Laursen PB, Stanley J, Kilding AE, Buchheit M. Training adaptation and heart rate variability in elite endurance athletes: opening the door to effective monitoring. Sports Med. 2013;43(9):773-781. doi:10.1007/s40279-013-0071-8 PubMed

39. Buchheit M, Al Haddad H, Laursen PB, Ahmaidi S. Effect of body posture on postexercise parasympathetic reactivation in men. Exp Physiol. 2009;94(7):795-804. doi:10.1113/expphysiol.2009.048041 PubMed

40. Aubry A, Hausswirth C, Louis J, Coutts AJ, Buchheit M, Le Meur Y. The development of functional overreaching is associated with a faster HR recovery in endurance athletes. PLoS One. 2015. file:///C:/Users/ Robin/Downloads/PLoS ONE 2015.pdf. Accessed October 6, 2015.

41. Daanen HAM, Lamberts RP, Kallen VL, Jin A, Van Meeteren NLU. A systematic review on heart-rate recovery to monitor changes in training status in athletes. Int J Sports Physiol Perform. 2012;7(3):251-260. PubMed doi:10.1123/ijspp.7.3.251

42. Andersson H, Raastad T, Nilsson J, Paulsen G, Garthe I, Kadi F. Neuromuscular fatigue and recovery in elite female soccer: effects of active recovery. Med Sci Sports Exerc. 2008;40(2):372-380. doi:10.1249/ mss.0b013e31815b8497 PubMed

43. Ispirlidis I, Fatouros IG, Jamurtas AZ, et al. Time-course of changes in inflammatory and performance responses following a soccer game. Clin J Sport Med. 2008;18(5):423-431. doi:10.1097/ JSM.0b013e3181818e0b PubMed

44. Magalhães J, Rebelo A, Oliveira E, Silva JR, Marques F, Ascensão A. Impact of Loughborough Intermittent Shuttle Test versus soccer match on physiological, biochemical and neuromuscular parameters. Eur J Appl Physiol. 2010;108(1):39-48. doi:10.1007/s00421-009-1161-z PubMed 
45. Fatouros IG, Chatzinikolaou A, Douroudos II, et al. Time-course of changes in oxidative stress and antioxidant status responses following a soccer game. J Strength Cond Res. 2010;24(12):3278-3286. doi:10.1519/JSC.0b013e3181b60444 PubMed

46. Twist $\mathrm{C}$, Highton J. Monitoring fatigue and recovery in rugby league players. Int J Sports Physiol Perform. 2013;8(5):467-474. PubMed doi:10.1123/ijspp.8.5.467

47. Nédélec M, McCall A, Carling C, Legall F, Berthoin S, Dupont G. Recovery in soccer: part I-post-match fatigue and time course of recovery. Sports Med. 2012;42(12):997-1015. doi:10.2165/11635270000000000-00000 PubMed

48. Malone JJ, Murtagh C, Morgans R, Burgess D, Morton JP, Drust B. Countermovement jump performance is not affected during an inseason training microcycle in elite youth soccer players. J Strength Cond Res. 2015;29(3):752-757. doi:10.1519/JSC.0000000000000701 PubMed

49. Buchheit M, Mendez-Villanueva A, Quod MJ, Poulos N, Bourdon P. Determinants of the variability of heart rate measures during a competitive period in young soccer players. Eur J Appl Physiol. 2010;109(5):869-878. doi:10.1007/s00421-010-1422-x. PubMed

50. Gibson NE, Boyd AJ, Murray AM. Countermovement jump is not affected during final competition preparation periods in elite rugby sevens players. J Strength Cond Res. 2016;30(3):777-783. doi:10.1519/JSC.0000000000001156 PubMed

51. Gathercole R, Sporer B, Stellingwerff T, Sleivert G. Alternative countermovement-jump analysis to quantify acute neuromuscular fatigue. Int J Sports Physiol Perform. 2015;10(1):84-92. doi:10.1123/ ijspp.2013-0413. PubMed

52. Cormack SJ, Newton RU, McGuigan MR, Cormie P. Neuromuscular and endocrine responses of elite players during an Australian rules football season. Int J Sports Physiol Perform. 2008;3(4):439-453. PubMed doi:10.1123/ijspp.3.4.439

53. Whiteley R. "Moneyball" and time to be honest about preseason screening: it is a sham making no inroads on the 1 billion dollar injury costs in baseball. Br J Sports Med. 2016;50(14):835-836. doi:10.1136/ bjsports-2014-094541. PubMed

54. Mohr M, Draganidis D, Chatzinikolaou A, et al. Muscle damage, inflammatory, immune and performance responses to three football games in 1 week in competitive male players. Eur J Appl Physiol. 2016;116(1):179-193. doi:10.1007/s00421-015-3245-2 PubMed

55. Paul DJ, Nassis GP, Whiteley R, Marques JB, Kenneally D, Chalabi $\mathrm{H}$. Acute responses of soccer match play on hip strength and flexibility measures: potential measure of injury risk. J Sports Sci. 2014;32(13):1318-1323. doi:10.1080/02640414.2014.927069. PubMed

56. McLellan CP, Lovell DI, Gass GC. Markers of postmatch fatigue in professional Rugby League players. J Strength Cond Res. 2011;25(4):1030-1039. doi:10.1519/JSC.0b013e3181cc22cc PubMed

57. Thorpe R, Sunderland C. Muscle damage, endocrine, and immune marker response to a soccer match. J Strength Cond Res. 2012;26(10):27832790. PubMed doi:10.1519/JSC.0b013e318241e174

58. Baird MF, Graham SM, Baker JS, Bickerstaff GF. Creatine-kinaseand exercise-related muscle damage implications for muscle performance and recovery. J Nutr Metab. 2012;2012:960363. doi:10.1155/2012/960363

59. Tidball JG. Inflammatory processes in muscle injury and repair. $A m$ J Physiol Regul Integr Comp Physiol. 2005;288(2):R345-R353. doi:10.1152/ajpregu.00454.2004. PubMed

60. Morgans R, Owen A, Doran D, Drust B, Morton JP. Prematch salivary secretory immunoglobulin a in soccer players from the 2014 World Cup qualifying campaign. Int J Sports Physiol Perform. 2015;10(3):401-403. http://dx.doi.org/10.1123/ijspp.2014-0046
61. Morgans R, Orme P, Anderson L, Drust B, Morton JP. An intensive winter fixture schedule induces a transient fall in salivary IgA in English Premier League soccer players. Res Sports Med. 2014;22(4):346354. doi:10.1080/15438627.2014.944641. PubMed

62. McCall A, Davison M, Carling C, Buckthorpe M, Coutts AJ, Dupont G. Can off-field "brains" provide a competitive advantage in professional football? Br J Sports Med. 2016;50(12):710-712. doi:10.1136/ bjsports-2015-095807. PubMed

63. Coutts AJ. Working fast and working slow: the benefits of embedding research in high performance sport. Int J Sports Physiol Perform. 2016;11(1):1-2. doi:10.1123/IJSPP.2015-0781 PubMed

64. Atkinson G, Nevill AM. Statistical methods for assessing measurement error (reliability) in variables relevant to sports medicine. Sports Med. 1998;26(4):217-238. PubMed doi:10.2165/00007256-199826040-00002

65. Atkinson G. Does size matter for sports performance researchers? J Sports Sci. 2003;21(2):73-74. doi:10.1080/0264041031000071038. PubMed

66. Jones PW, Beeh KM, Chapman KR, Decramer M, Mahler DA, Wedzicha JA. Minimal clinically important differences in pharmacological trials. Am J Respir Crit Care Med. 2014;189(3):250-255. doi:10.1164/ rccm.201310-1863PP PubMed

67. Rai SK, Yazdany J, Fortin PR, Aviña-Zubieta JA. Approaches for estimating minimal clinically important differences in systemic lupus erythematosus. Arthritis Res Ther. 2015;17:143. doi:10.1186/s13075015-0658-6 PubMed

68. Hopkins WG. sportsci.org. http://sportsci.org/jour/04/wghtests.htm

69. Batterham AM, Atkinson G. How big does my sample need to be?: a primer on the murky world of sample size estimation. Phys Ther Sport. 2005;6:153-163. doi:10.1016/j.ptsp.2005.05.004

70. Kempton T, Sirotic A, Coutts A. Between match variation in professional rugby league competition. J Sci Med Sport. 2014;17:404-407. PubMed doi:10.1016/j.jsams.2013.05.006

71. Cook JA, Hislop J, Adewuyi TE, et al. Assessing methods to specify the target difference for a randomised controlled trial: DELTA (Difference ELicitation in TriAls) review. Health Technol Assess. 2014;18(28):vvi, 1-175. doi:10.3310/hta18280. PubMed

72. Hemingway H, Croft P, Perel P, et al. Prognosis research strategy (PROGRESS) 1: a framework for researching clinical outcomes. BMJ. 2013;346:e5595. PubMed doi:10.1136/bmj.e5595

73. Finch CF, Marshall SW. Let us stop throwing out the baby with the bathwater: towards better analysis of longitudinal injury data. Br J Sports Med. 2016;50(12):712-715. doi:10.1136/bjsports-2015-094719. PubMed

74. Wyrwich KW, Tierney WM, Wolinsky FD. Further evidence supporting an SEM-based criterion for identifying meaningful intraindividual changes in health-related quality of life. J Clin Epidemiol. 1999;52(9):861-873. PubMed doi:10.1016/S0895-4356(99)00071-2

75. Bland JM, Altman DG. Measuring agreement in method comparison studies. Stat Methods Med Res. 1999;8(2):135-160.. PubMed doi:10.1191/096228099673819272

76. Bland JM, Altman DG. Applying the right statistics: analyses of measurement studies. Ultrasound Obstet Gynecol. 2003;22(1):85-93. doi:10.1002/uog.122. PubMed

77. Cohen J. Statistical Power Analysis for the Behavioral Sciences. 2nd ed. Hillsdale, NJ: L. Erlbaum; 1988.

78. Batterham AM, Hopkins WG. Making meaningful inferences about magnitudes. Int J Sports Physiol Perform. 2006;1(1):50-57. PubMed doi:10.1123/ijspp.1.1.50

79. McLean BD, Coutts AJ, Kelly V, McGuigan MR, Cormack SJ. Neuromuscular, endocrine, and perceptual fatigue responses during different length between-match microcycles in professional rugby 
league players. Int J Sports Physiol Perform. 2010;5(3):367-383. PubMed doi:10.1123/ijspp.5.3.367

80. Montgomery PG, Hopkins WG. The effects of game and training loads on perceptual responses of muscle soreness in Australian football. Int J Sports Physiol Perform. 2013;8(3):312-318. PubMed doi:10.1123/ ijspp.8.3.312

81. Drew MK, Finch CF. The relationship between training load and injury, illness and soreness: a systematic and literature review. Sports Med. 2016;46(6):861-883. doi:10.1007/s40279-015-0459-8. PubMed

82. Foster C, Florhaug JA, Franklin J, et al. A new approach to monitoring exercise training. J Strength Cond Res. 2001;15(1):109-115. PubMed

83. Williams J, Andersen M. Psychosocial antecedents of sport injury and interventions for risk reduction. In: Handbook of
Sport Psychology. 3rd ed. New York, NY: Wiley; 2007:379-403. doi:10.1002/9781118270011.ch17

84. Johnson U, Ivarsson A. Psychological predictors of sport injuries among junior soccer players. Scand J Med Sci Sports. 2011;21(1):129_ 136. doi:10.1111/j.1600-0838.2009.01057.x. PubMed

85. Brink MS, Frencken WGP, Jordet G, Lemmink KA. Coaches' and players' perceptions of training dose: not a perfect match. Int J Sports Physiol Perform. 2014;9(3):497-502. doi:10.1123/ijspp.2013-0009. PubMed

86. Laux P, Krumm B, Diers M, Flor H. Recovery-stress balance and injury risk in professional football players: a prospective study. $J$ Sports Sci. 2015;33(20):2140-2148. doi:10.1080/02640414.2015.1 064538. PubMed 


\section{Erratum: Thorpe, Atkinson, Drust, and Gregson (2017)}

Atkinson is with the Health and Social Care Inst, Teesside University, Teesside, UK, and Drust and Gregson are with the Research Inst for Sport and Exercise Sciences, Liverpool John Moores University, Liverpool, UK. Their affiliations were incorrectly switched with each other in the original online-only version. This online version has been corrected. 